\title{
A NOVEL PLANAR SCISSOR STRUCTURE TRANSFORMING BETWEEN CONCAVE AND CONVEX CONFIGURATIONS
}

\author{
M. YAR ${ }^{1}$, K. KORKMAZ1, G. KIPER ${ }^{1}$, F. MADEN ${ }^{2}$, Y. AKGÜN ${ }^{3} \&$ E. AKTAŞ ${ }^{1}$ \\ ${ }^{1}$ Izmir Institute of Technology, Turkey. \\ ${ }^{2}$ Yaşar University, Turkey. \\ ${ }^{3}$ Gediz University, Turkey.
}

\begin{abstract}
In this paper, a novel two-dimensional scissor structure that transforms between concave and convex configurations is presented. The structure is designed by a method of assembling kite or anti-kite loops in the flat configuration. Angulated units are generated from the assembled loops. Finally, a new angulated scissor unit is introduced in order to design the novel scissor structure.

Keywords: angulated units, anti-kite loops, kite loops, scissor structure.
\end{abstract}

\section{INTRODUCTION}

Deployable scissor structures transform from a compact stowed configuration to an expanded functional configuration, which provide them to be used in portable or temporary applications. Since they are adaptable structures, they can be integrated either to a site as a deployable bridge to respond changing transportation requirements or to a building as a deployable roof for changing weather conditions.

Scissor mechanisms are most preferred units for deployable systems and there are various studies about deployable structures constructed with them. First, architectural scissor structure in the literature was designed by Spanish architect Emilio Perez Pinero [1] in 1961. This structure was further developed by Escrig and Valcarcel as in the form of new spherical grid structures that are composed of two-way and three-way scissors with several connection details [2-5]. In addition to these developments, Hoberman [6] proposed a novel concept composed of angulated elements that led to design radially deploying closed loop structures. In 1997, You and Pellegrino [7] discovered generalised angulated elements to be used as a building block. They also discovered multi-angulated rod that reduced the number of components of the structure and the complexity of its joints.

All 2D or 3D deployable scissor structures are closed loops mechanisms. There are three types of scissor units noted in the literature: translational, polar and angulated (Fig. 1). These units, connected to build deployable structures, form similar rhombus, parallelogram or kite loops. So, another type of classification due to the loops can be obtained when the scissor units are assembled. In an MIT lecture note, Hoberman [8] introduces a method for the geometric construction of expanding polygons with angulated elements by assembling equal or unequal rhombuses.

In this paper, a new deployable scissor structure is presented. It is designed by assembling kite and anti-kite loops. The new two structures have two different stowed configurations by bending upward and downward. The layout of the paper is as follows. Section 2 reviews the basic scissor units with respect to their loop types. Section 3 introduces an alternative design approach that deals with the assemblies of identical kite and anti-kite loops to form a novel deployable planar structure. Two different cases of this approach are demonstrated in Solidworks. 


\section{BASIC SCISSOR UNITS}

Planar scissor structural mechanisms consist of different types of scissor units. A scissor unit is composed of two straight or angulated bars that are connected to each other by a revolute joint on the point of their axis. This revolute joint, also called as the scissor hinge, allows scissor unit to move with a single degree of freedom (DOF). The position of the scissor hinge on the bars and the shape of the bars determine the type of the scissor unit.

There is an imaginary axis between the upper end point of one bar and the bottom end point of the other bar, which is called a unit line. The most considerable characteristic of the translational units is that their unit lines remain parallel to each other during the deployment and stowing process. It is observed that the loops of the deployed structure are equilateral parallelograms (rhombus) (Fig. 1a).

If the unit lines of a scissor unit are no longer parallel to each other, the scissor unit is a polar or an angulated scissor unit. A polar scissor unit is obtained by connecting two straight bars with scissor hinges away from the midpoints of the bars (Fig. 1b). The segment angle $(\alpha)$ varies, but the structure draws a concentric circle during the deployment. The loops of the deployed structure are deltoid (kite) (Fig. 1b). In accordance with the size of polar units, loops can form different kites [9]. Hoberman's angulated unit is obtained by connecting two identical angulated bars, which have a kink with an angle $(\Psi)$ instead of straight bars [10]. The structure draws a concentric circle. However, the segment angle $(\alpha)$ between two unit lines is constant during the deployment, whereas it varies in polar units [9]. It is observed that the loops of the structure are rhombuses (Fig. 1c).

In addition to the Hoberman's angulated scissor unit, You and Pellegrino [7] discovered 'generalized angulated elements'. The first type of generalized angulated units is Equilateral Angulated Element formed by angulated rods with equal semi-length but not necessarily equal kink angles: $|A E|=|D E|,|C E|=|B E|, \phi \neq \Psi$. As a special case, Hoberman's angulated elements are obtained by equal kink angles. In order to obtain radial deployment with constant segment angles, each closed loop in this system must be a rhombus (Fig. 2) [7].
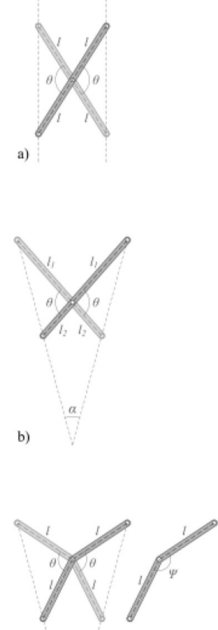
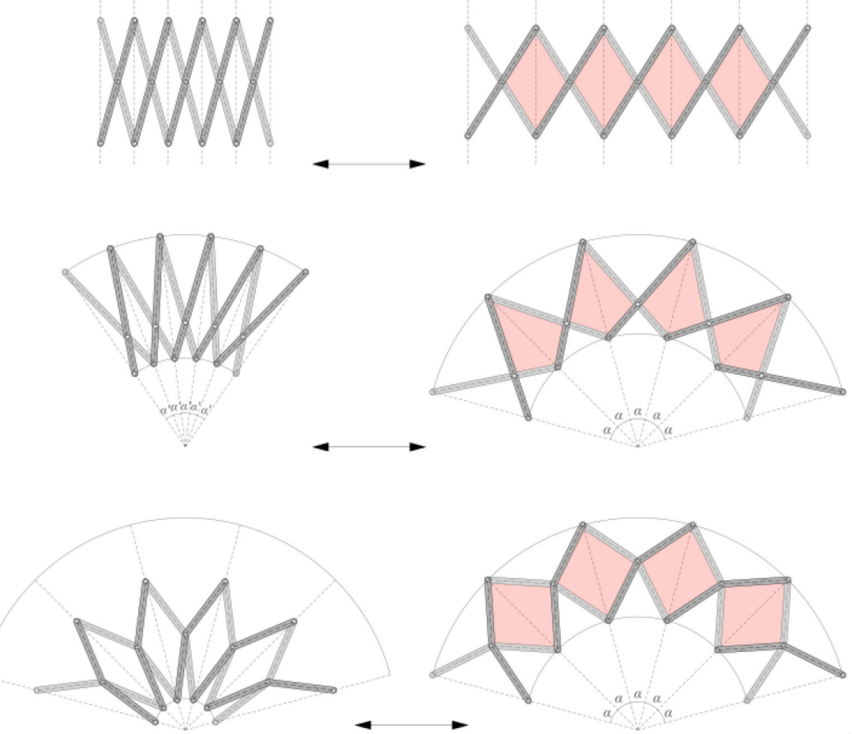

Figure 1: Basic scissor units and their loop types. 
The second type of generalized angulated unit (Type II) is the Similar Angulated Element formed by angulated rods with proportional semi-lengths and having equal kink angles: $|A E| /|D E|=|C E| /|B E|, \phi=\Psi$. In order to be deployable, each closed loop must be a parallelogram (Fig. 3) [7].

Aforementioned deployable scissor mechanisms are composed of one of the three types of loops that are parallelogram, rhombus or kite loops. In this study, it is aimed to propose a novel deployable structure with angulated scissor units forming kite or anti-kite loops which has ability to transform between concave and convex configurations.

\section{METHOD OF ASSEMBLING IDENTICAL KITE AND ANTI-KITE LOOPS}

When deployable structures formed by angulated scissor units are investigated, the most important characteristic of the units is detected as that the angles between unit lines remain constant during deployment process. In order to obtain constant segment angles, angulated units are assembled to each other in such a way that each closed loops are a parallelograms or a rhombus. Hoberman [8] presents a method to obtain the angulated scissors. It is based on assembling identical or different rhombuses to each other and then generating angulated scissor units. In this section, this paper proposes a structure that can transform between concave and convex configurations. In order to obtain the structure, Hoberman's construction method is used to assemble the identical kite or anti-kite loops.

In Fig. 4, two kite loops are assembled in flat configuration where unit lines are parallel. After the assembly, angulated scissor unit is drawn on the kites, which has equal semi-lengths and equal kink angles.

In Figs 5 and 6, the transformation capability of a single DOF deployable structure obtained by assembling the kite loops is shown. In flat configuration, unit lines are parallel and the curvature is equal to zero. By increasing the angle $\theta$ of only one kite loop, the structure bends upward. Unit lines intersect and define a center which translates on $+y$ axis because the

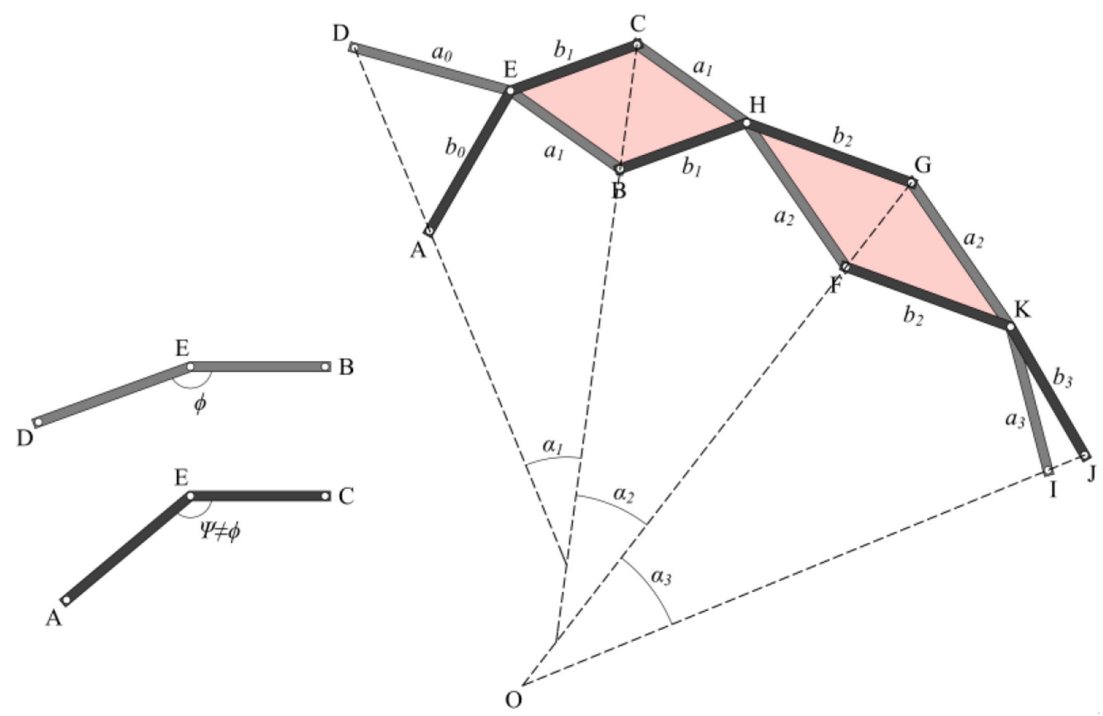

Figure 2: Assembly of Equilateral Angulated Elements forming rhombus loops with constant segment angles $\alpha_{1}, \alpha_{2}, \alpha_{3}$. 


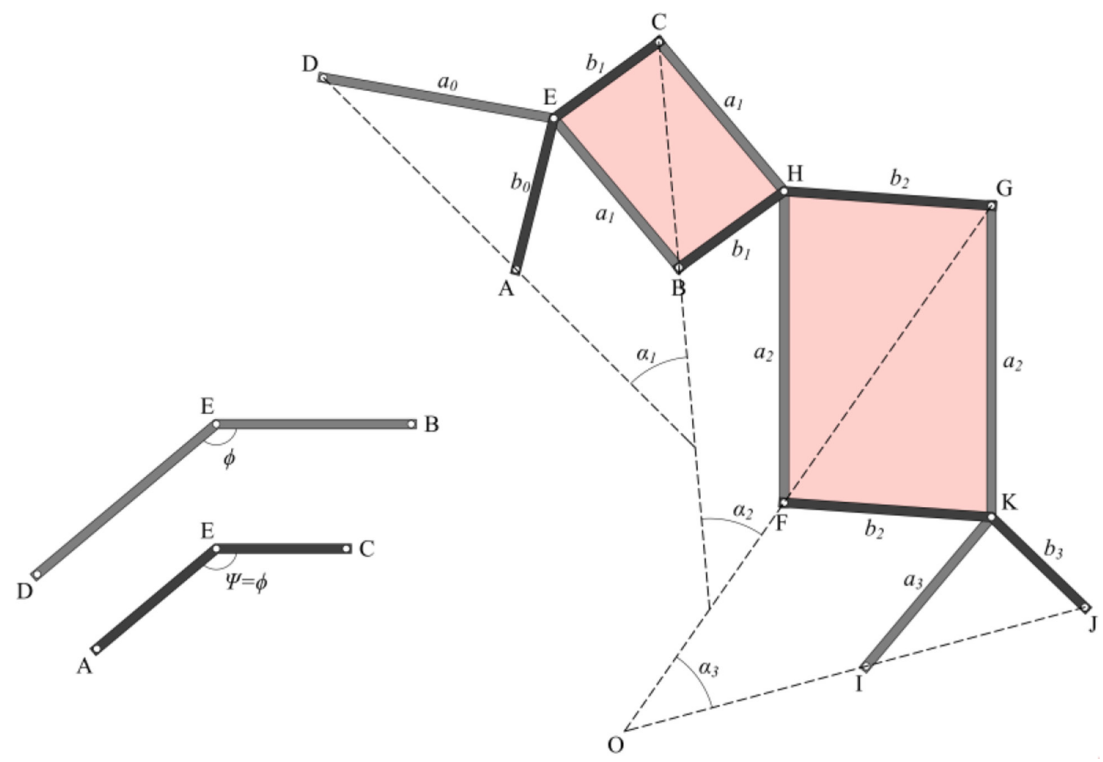

Figure 3: Assembly of Similar generalized angulated elements forming parallelogram loops with constant segment angles $\alpha_{1}, \alpha_{2}, \alpha_{3}$.

segment angles vary during the deployment. The radius decreases, i.e. the curvature increases while the structure bends up. Meanwhile, the kite loops transform into anti-kite loops.

On the other hand, by decreasing the angle $\theta$ of only one kite loop, the structure bends downward (Fig. 6). Unit lines intersect and define a center, which translates on $-y$-axis

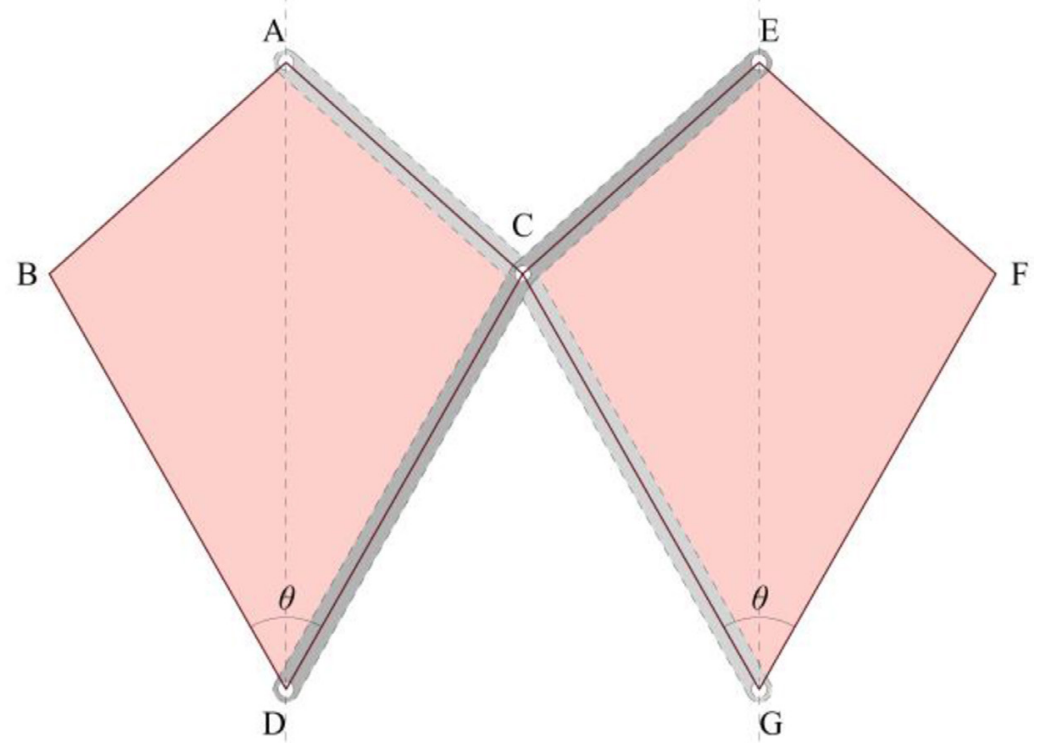

Figure 4: Assembly of kite loops and generating angulated scissors. 


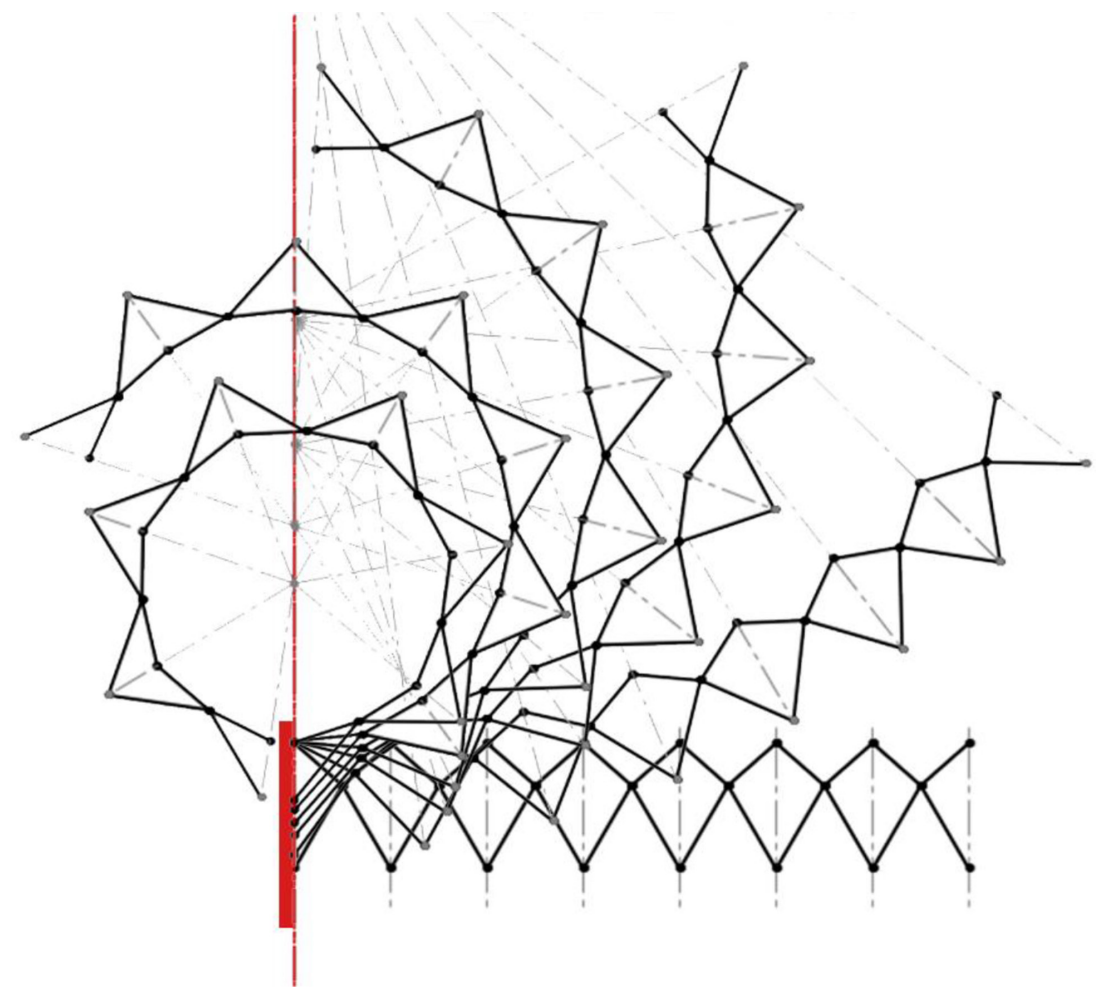

Figure 5: From flat configuration to bended upward configuration.

because segment angles vary during the deployment. The radius decreases, i.e. the curvature increases while bending down.

Same procedure can also be applied to anti-kite loops as it is seen in Fig. 7. Anti-kite loops are assembled in flat configuration where the unit lines are parallel. After the assembly,

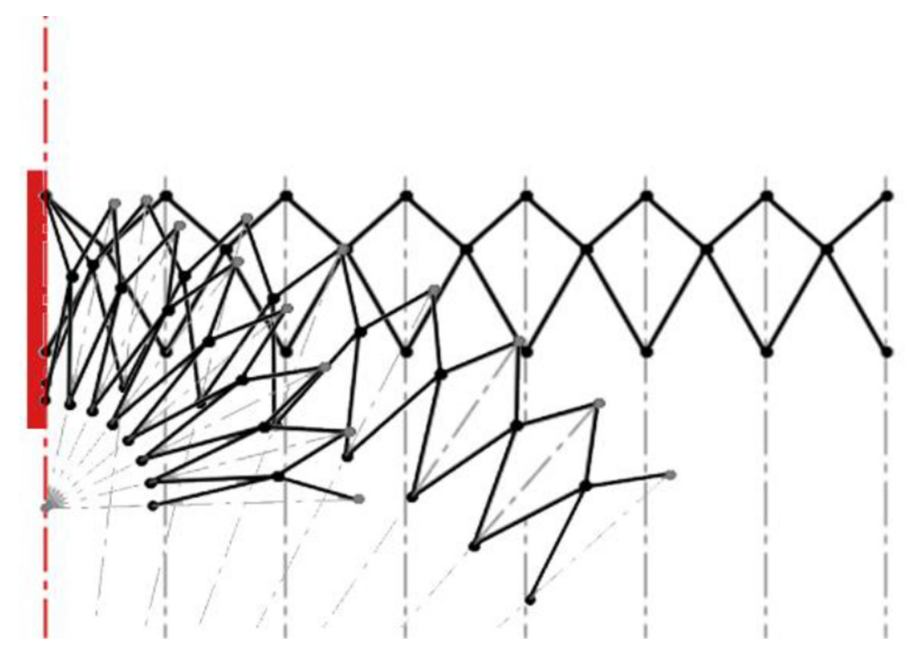

Figure 6: From flat configuration to bended downward configuration. 
angulated scissor unit is drawn on the anti-kites, which has equal semi-lengths and equal kink angles.

In Figs 8 and 9, the transformation capability of a single DOF deployable structure obtained by assembling the anti-kite loops is shown. In flat configuration, the unit lines are parallel and

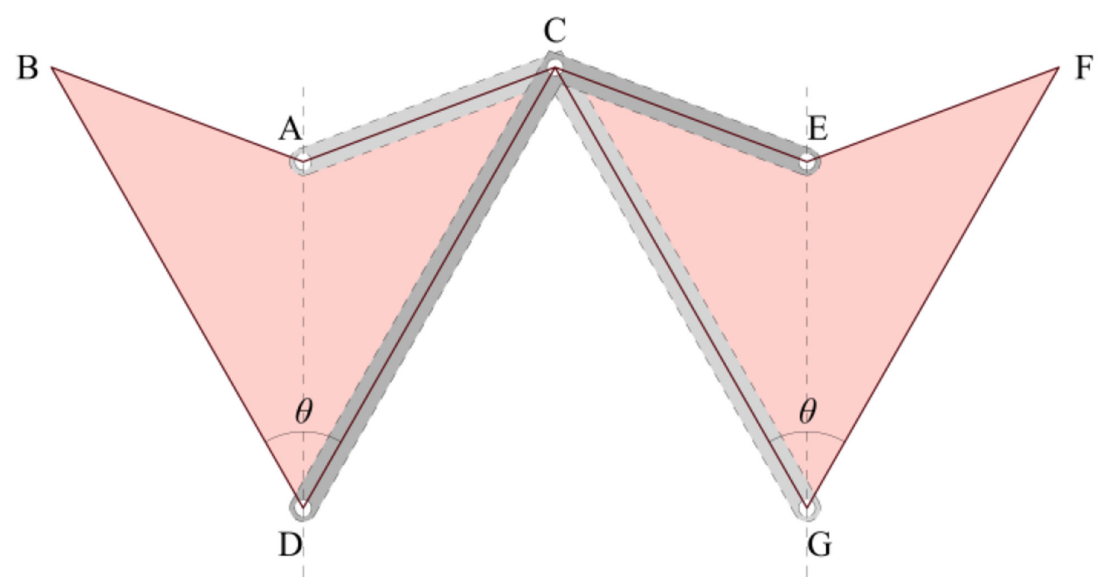

Figure 7: Assembly of anti-kite loops and generating angulated scissor unit.

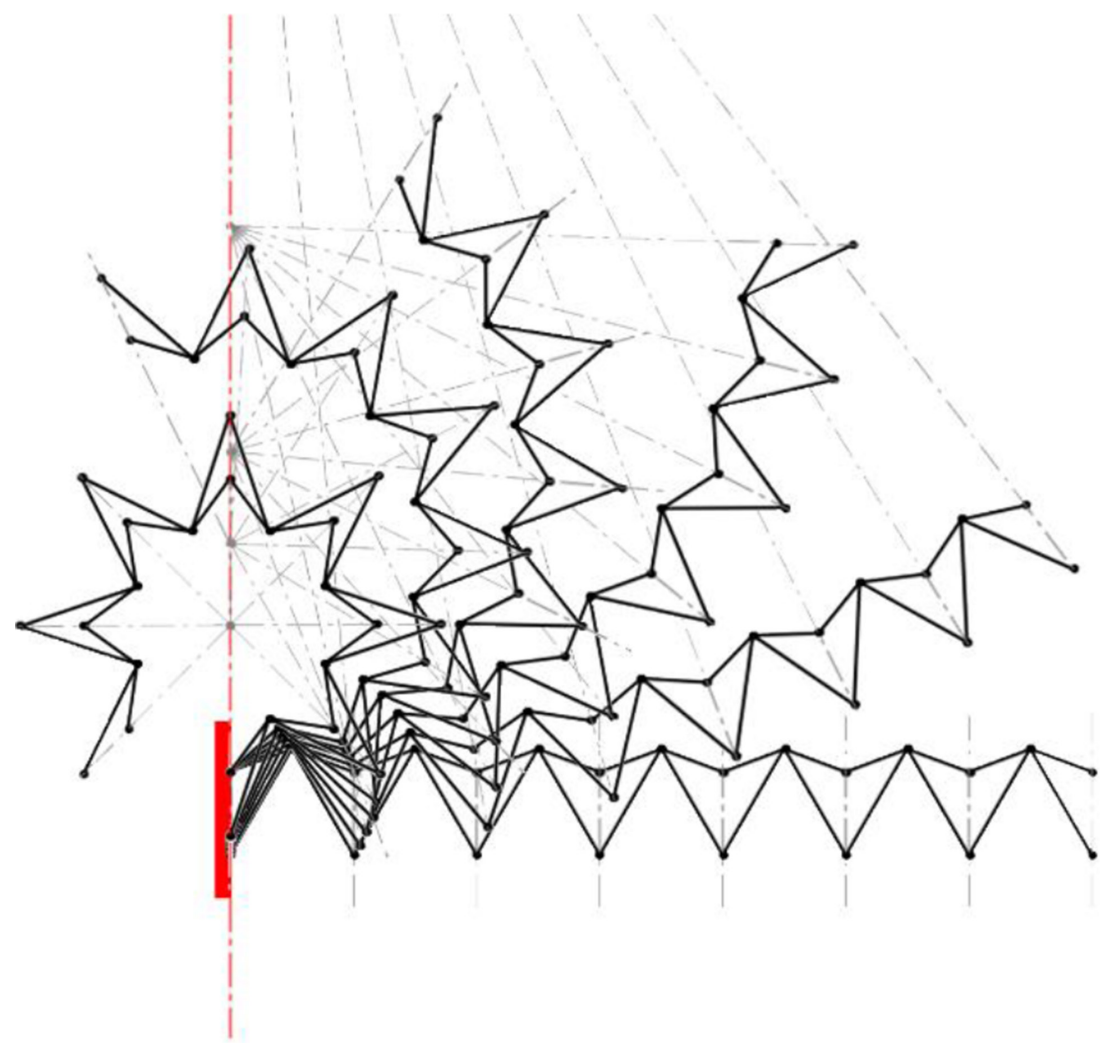

Figure 8: From flat configuration to bended upward configuration. 


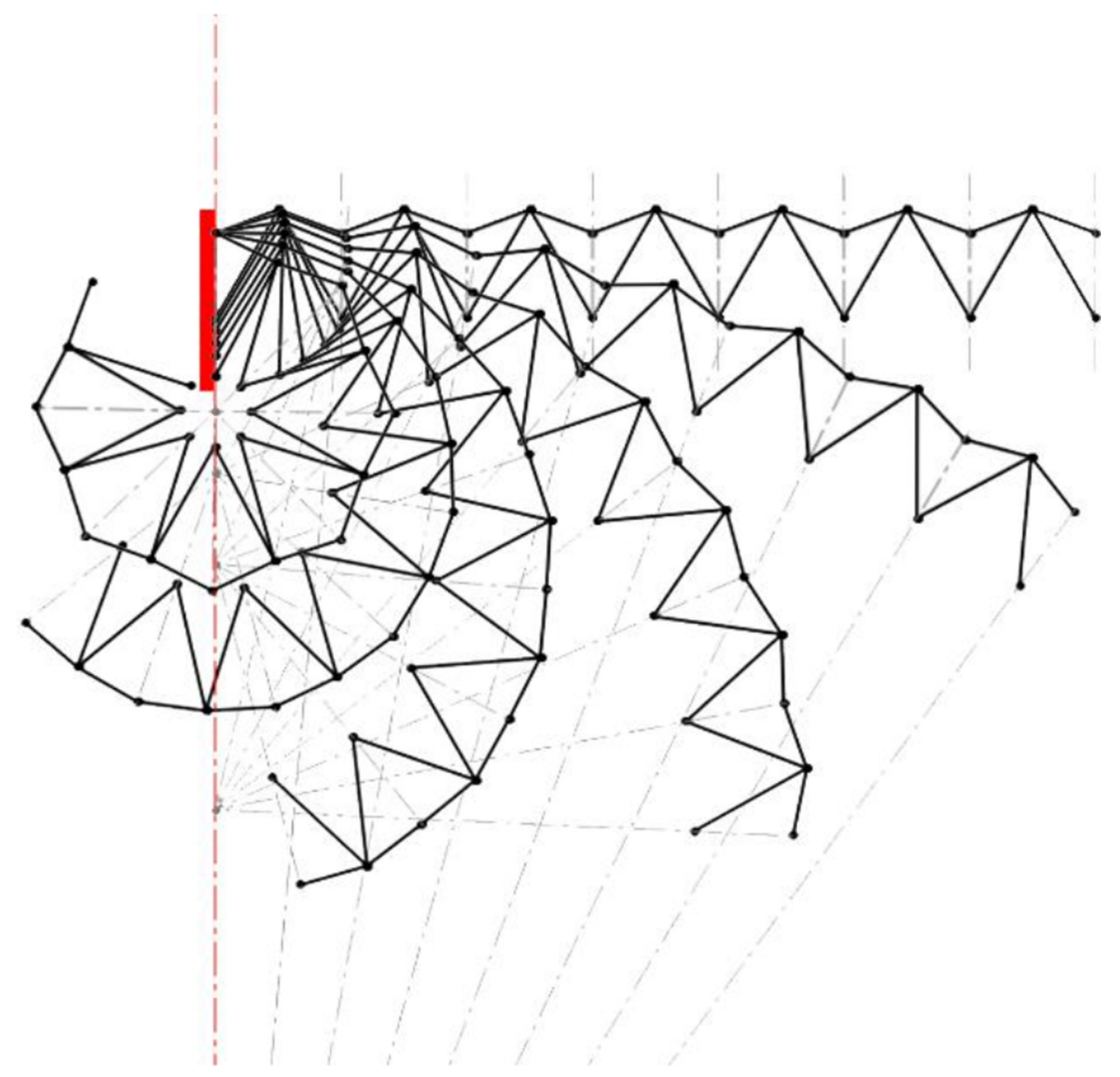

Figure 9: From flat configuration to bended downward configuration.

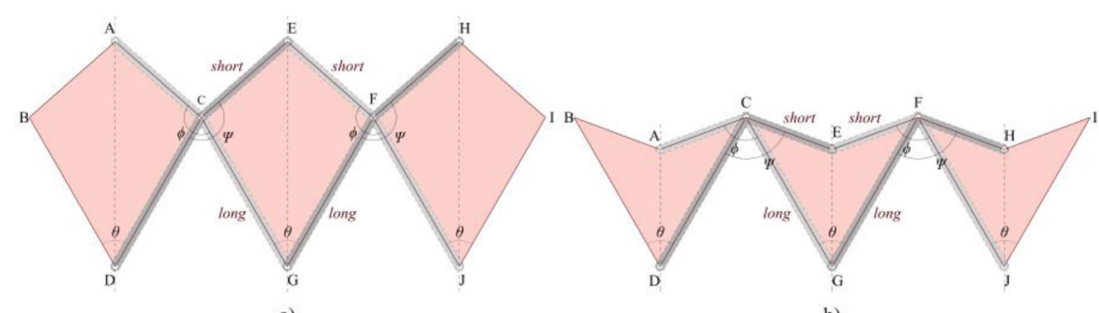

a)

b)

Figure 10: A novel scissor unit obtained by assembling kite and anti-kite loops.

the curvature is equal to zero. Conversely, this time by decreasing the angle $\theta$ of only one kite loop, the structure bends upward. Unit lines intersect and define a center which translates on $+y$ axis, because the segment angles vary during deployment. The radius decreases, i.e. the curvature increases while bending up.

On the other hand, by increasing the angle $\theta$ of only one anti-kite loop, the structure bends downward (Fig. 8). Unit lines intersect and define a center, which translates on $-y$-axis, because the segment angles vary during deployment. The radius decreases, i.e. the curvature increases while bending down. Meanwhile, the anti-kite loops transform into kite loops. 
After the geometric construction of two novel deployable structures, a new scissor unit is developed which is obtained by assembling identical kite loops. As seen in Fig. 10a and b, the new scissor unit satisfies the following conditions:

$$
|A C|<|D C|,|C G|>|C E|, \phi=\Psi
$$

Semi-bars on both sides of a unit line are one short and one long semi-bars. There is mirror symmetry about the unit line.

\section{CONCLUSION}

In this paper, a novel two-dimensional single DOF scissor structure has been introduced. A new type of angulated scissor unit has been developed and a method of assembling kite or anti-kite loops has been used in order to design the scissor structure. The new unit has been generated from the assembled loops. The proposed structure bends upward and downward simultaneously, i.e. it transforms between concave to convex configurations.

\section{ACKNOWLEDGMENT}

This project has received funding from the European Union's Horizon 2020 research and innovation programme under the Marie Skłodowska-Curie grant agreement No 689983.

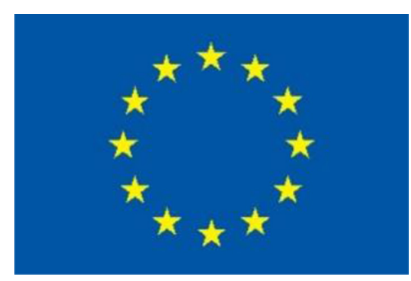

\section{REFERENCES}

[1] Pinero, E., Project for a mobile theatre. Architectural Design, 12, p. 570, 1961.

[2] Escrig, F., Expandable space frame structures. Proceedings of the 3rd International Conference on Space Structures, ed H. Nooshin, Elsevier Science Publishers: London, pp. 845-850, 1984.

[3] Escrig, F., Expendable space structures. International Journal of Space Structures, 1(2), pp. 79-91, 1985.

[4] Escrig, F. \& Valcarcel, J., Great size umbrellas with expandable bar structures. Proceedings of the 1st International Conference on Lightweight Structures in Architecture, University of New South Gales: Sydney, pp. 676-681, 1986.

[5] Escrig, F. \& Valcarcel, J., Curved expandable space grids. Proceedings of the International Conference on the Design and Construction of Non-Conventional Structures, Edinburg, Civil Comp Press, pp. 157-166, 1987. http://dx.doi.org/10.4203/ccp.7.18.1

[6] Hoberman, C., Reversibly expandable doubly-curved truss structure, US Patent 4.942.700, 1990.

[7] You, Z. \& Pellegrino, S., Expandable bar structures. International Journal of Solid Structures, 34(15), pp. 1825-1847, 1997. http://dx.doi.org/10.1016/S0020-7683(96)00125-4 
[8] Hoberman, C., Mechanical invention through computation - expanding structures - 2D, MIT Class 6.S080 online course material, available at: http://courses.csail.mit.edu/6. S080/lectures/02_all.pdf

[9] Maden, F., Korkmaz, K. \& Akgün, Y., A review of planar scissor structural mechanisms: geometric principles and design methods. Architectural Science Review, 54(3), pp. 246-257, 2011.

http://dx.doi.org/10.1080/00038628.2011.590054

[10] Jensen, F., Concepts for retractable roof structures, PhD Thesis, University of Cambridge, 2004. 